\title{
Prevention of depression and suicide in Primary Health Care settings: a systematic review of the evidence
}

\author{
By Stina K. Söderqvist and Ellenor Mittendorfer-Rutz
}

This article systematically reviews the literature concerning educational training programmes for primary health care staff aiming to improve detection and treatment of depression and thereby reducing suicidal behaviour. PubMed, PsychINFO, York CRD and Cochrane library searches were conducted for studies targeting on detection and prevention of depression and/or suicide prevention. All programmes except one, succeeded in significantly improving the detection of suicidal ideation and/or reducing suicidal behaviour. Findings from programmes solely aiming to improve detection of depression were more discrepant. Differences between the interventions are highlighted. A number of issues such as the consideration of needs assessments, gender differences and the sustainability of effects are discussed as important prerequisites for effective prevention programmes.

\section{Introduction}

Suicide is increasing world-wide and is now one of the three most common causes of death in the age group of 15-44 (SUPRE, WHO, 2007; MittendorferRutz et al., 2004). This increasing problem warrants the development of tailormade prevention strategies. To date, evidence on a wide range of intervention methods both within Public Health and the Health care sector is emerging (Mann et al., 2005; Mittendorfer-Rutz et al., 2004a). The public health strategies include restricting access to lethal means such as weapons and pesticides; responsible media reporting and educational programmes for the public, and populations at risk. The strategies focusing on health care include improved medical care, improved follow-up of patients with a suicidal history and education of general practitioners (GPs) in detecting and treating depression. A recently published review of suicide prevention strategies by Mann and colleagues (2005), concludes that education of primary care physicians and gatekeepers as well as interventions restricting access to lethal means both show promising results in reducing suicide rates (Mann et al., 2005).

\section{Rationale for educational interventions} at a primary health care level

Even though suicidal behaviour has a multifactorial ethiology, the majority of suicides are committed by people who suffer from some kind of mental illness. Lönnqvist et al. (1995) carried out psychological autopsies on all suicides committed in Finland during one year (a total of 1397 cases) and found that 93\% of these cases could be diagnosed with some kind of psychiatric disorder.
Out of these $66 \%$ were diagnosed as having suffered from depression. In a more recent review the same authors estimated that 29-88\% of suicide victims were suffering from depression (Lönnqvist, 2000).

Although $60-80 \%$ of cases of depression can be successfully treated (by medication and/or other types of therapy) the WHO estimates that only around $25 \%$ of cases are offered sufficient treat-ment. Lack of trained caregivers, lack of resources, low help-seeking behaviour and social stigma related to the disorder are identified as possible explanations (WHO, 2007).

An international review by Luoma et al. from 2002 suggests that a majority of suicide victims (approximately 75\%) were in contact with the primary health care within the year prior to suicide; while in contrast, only around $30 \%$ were in contact with the mental health service during that same period. This suggests that the primary health care is an appropriate arena in order to reach groups at risk. By improving the detection of depression and suicidal tendencies in such patients, suicidal outcomes should be able to be prevented.

\section{Aims and questions}

The primary question being asked in this review is whether educational programmes targeting primary health care staff can be effective in preventing suicide and depression. Outcomes that are of interest for this purpose include changes in rates of completed suicide and suicide attempts as well as the reported occurrence of suicidal thoughts and depressive symptoms among patients. How various programmes are perceived by the participating staff and the consequences on their practice, is also of interest and will be discussed where such data is available.

\section{Material and methods}

A systematic review of the literature was done using the search engines PubMed, PsychINFO, York CRD and the Cochrane library. Searches were carried out with the following terms: "suicide prevention" (alone),"primary health care" and "general practitioners", each combined in separate searches with the terms "suicide prevention", "depression prevention" and "depression detection". Manual searches of reference lists of relevant articles and reviews were also carried out.

\section{Inclusion criteria}

Articles on interventions within the primary health care services aiming to improve detection and care for patients with suicidal ideation and/or depression were included. The searches were limited to include only articles written in English. There were no limitations for country of origin or year of publication.

\section{Exclusion criteria}

Articles that focused on specific treatments or screening methods were excluded. Similarly, interventions focusing on certain patient groups such as patients with alcohol abuse were also excluded, as these tend to focus on specific treatment strategies and/or specialist care.

\section{Results}

All interventions reviewed here aimed at improving detection and/or management of depression in primary health care practices. Some had an additional interest on reducing rates of suicidal behaviour. Interventions including suicidal behaviour as an outcome measure are presented separately from those with outcome measures limited to depression. 
Table 1: Interventions with suicidal behaviour included as outcome measures

\section{Author, region, target population}

Asarnow et al. (2005) US. Adolescents

Bruce et al. (2004)

Philadelphia and

Pittsburgh regions, US.

Elderly population

\section{Study design, type} of intervention

Randomised controlled trial (RCT). Educational intervention for GPs and care managers.

RCT. Intervention targeting on physician's knowledge and treatment management. Use of depression care managers.

Cohort, quasi-experimental. Educational programme for GPs.

Sweden.

General population

Hegerl et al. (2006).

Nuremberg, Germany.

General population

Nutting et al. (2005).

California, US.

General population.

Pfaff et al. (2001).

Tasmania, Victoria and Western Australia. Young patients.

Rutz et al. (1989a, 1989b, 1999, and 2001). Gotland, Sweden.

General population.

Szanto et al. (2007). Southwest Hungary. General population.

Cohort, quasi-experimental Intervention at 4 levels, of projects including training of either nurse care managers or a quality improvement intervention for care practices.

Cohort, pre-/posttest design. Educational programme for GPs on recognition of psychological distress and suicidal ideation in young people.

Cohort, quasi-experimental. Educational programme for GPs.

Cohort, quasi-experimental educational programme and establishment of a which one was training of GPs.

RCT, two different educational depression treatment clinic and telephone consultant service.

\section{Outcome measures}

Rates of suicidal behaviour at a 6 month follow-up.

Suicide ideation and symptoms of depression.

Suicide rates and prescription rates of antidepressants medication prior and during the intervention period.

Results

Reduction of suicide attempts and reported suicidal ideation, however not sign compared to a care as usual control group.

Sign reduction of suicidal ideation for patients with major depression only. Sign decreases of, and recovery from, depressive symptoms. Effects remained sign after 4 - but not 8 or 12 months.

Sign reduced suicide rates. Sign increase of antidepressants sales. Effects seen amongst women only.

Sign decrease of suicide attempts but no sign effect on completed suicides. suicide attempts during three years.

Rates of detected cases of suicidal ideation prior and post intervention and at a 6 month follow-up.

Rates of detected cases of suicidal ideation

Suicide rates and prescription rates of antidepressants.

Annual suicide rate and prescription use of antidepressants before, during and directly after the intervention period.
Both projects resulted in sign improvements in detection of suicidal ideation compared to usual care. Effects not sign at the follow-up.

Identification of patients with suicidal ideation increased by $130 \%$.

Sign. decrease in suicide rates and increase of prescription of antidepressants.

Sign decrease in suicide rate, compared to the rest of the county and country. Compared to a control region the effect was sign amongst women only. There was a sign increase of prescription rates. 


\section{Suicide prevention interventions in primary health care settings}

\section{General findings}

Six studies (with randomised control or cohort study design) were identified that evaluated interventions with rates of suicide, suicide attempts and/or suicidal ideation as outcome measures. Two studies that used detection of suicide ideation as outcome measures were also identified.

Effects on suicide, suicide attempts and/ or suicidal ideation

Rutz et al. (1989a, 1989b and 2001) summarise an intervention implemented on the island of Gotland in Sweden (with 58000 inhabitants), consisting of an educational programme in which general practitioners (GPs) participated in training seminars during two days. This was then repeated twice during a two year period. As a result this programme achieved a significant reduction in suicides (by 60\%), an effect that remained significant when compared to the national average of suicide rates. Henriksson and Isacsson (2006) replicated these findings of this so-called "Gotland study", in Jämtland, Sweden with a population of 135 854 (in 1995). Eighty-five GPs participated in at least one session of an annually repeated seminar (between the years of 1995 and 2002). Similarly to Rutz et al. (1989a) this intervention resulted in a reduction of regional suicide rates; from significantly above the national average prior intervention, to a level closer to the national average following the intervention (no longer significant). Following both studies an increase in prescription rates of antidepressant medication was observed. However only the Gotland study resulted in a change in measured attitudes amongst the participating GPs.

A similar recent programme was carried out in an area with high suicide rates in Hungary, including 28 GPs servicing 73 000 inhabitants (Szanto et al., 2007).

The five-year intervention was successful in decreasing rates of suicide, a change that was significant compared to national rates and local rates in the county.

Prescription rates of anti-depressant medication also increased significantly in the intervention area. However when compared to a control region the changes were significant amongst females only.

Nutting et al. (2005) rerport on two additional programmes that involved education of physicians regarding care of depressed patients (Nutting et al., 2005). One of them, QuEST, included 24 primary care physicians who participated in four conference session regarding guidelines for detection and treatment of depression. In addition, nurse care managers were educated (in an 8-hour training session) on how to inform depressed patients about treatment options as well as how to monitor patients' response to treatment. The nurse care managers then stood in direct contact with the patients. The second intervention, MHAP, focused on evidence based improvements of the depression care guidelines. Physicians, 179 in total, participated in a 4,5 hourlong interactive seminar on how these could be improved. In addition two quality improvement teams met for 16 hours, resulting in a proposal for practical improvements in care. Compared to a care as usual control group, both interventions led to significantly increased detection of suicide ideation in depressed patients. However, no effect was seen on referrals to psychiatric care or prescriptions of antidepressant medication, nor did the effects remain significant at a sixmonth follow-up.

A further study by Pfaff et al. (2001) consisted of a one-day training workshop where 23 GPs were taught about recognition and treatment of young suicidal patients. Compared to the assessment before intervention (including 203 patients), the precision in detecting suicide ideation improved with a reduction in false negative diagnoses and with a minimal increase in false positive cases (including assessments of 220 patients). As no measures of patient outcomes were included in any of these two latter studies it is difficult to judge their actual effectiveness in preventing suicide and depression.

Asarnow et al. (2005) focused on 211 young patients identified following screening for depression. GPs participating in the intervention group were provided with training and educational material on depression treatment. In addition expert leader teams adapted and implemented the intervention at each location. Care managers were also included to assist the primary care physicians with patient evaluation and medication. Compared to a care as usual control group the intervention resulted, at a six-month follow-up, in significantly decreased depressive symptoms, increased use of the mental health care (although not medication) and significantly lower rates of severe depression. Although effects were seen on decreased levels of suicide ideation and suicide attempts/self-harm, these effects were not significant compared with the care as usual control group. The PROSPECT intervention targeted on an elderly population at 20 primary care practices in three areas: New York City, Philadelphia, and Pittsburgh (Bruce et al., 2004). Depression care managers who had direct contact with patients and helped the GPs in recognising, treating and following up on depressed patients, were included in addition to guidelines tailored for care of elderly, available to the GPs. The intervention resulted in a significant decrease in reported suicidal ideation in patients with major depression. Furthermore the severity of other depressive symptoms was reduced and the response to depression treatment improved. No such effects were seen for patients with minor depression.

Finally the study by Hegerl et al. (2006) differs from the other studies described here due to its inclusion of additional public health strategies. Complementary to the training of GPs, the intervention included a public relations campaign, cooperation with community facilitators and support for different self-help activities. Results after the two-year long intervention period showed significantly reduced rates of suicide attempts compared to a control region; an effect particularly evident amongst high-risk suicide attempts. No significant difference was found between the intervention and control region with regard to rates of completed suicides. It is impossible to separate the level of effect the different activities might have had on these results and it should be noted that merely 20\% of GPs in the area participated in the training. 
Evidence for gender differences

Rutz et al. (e.g. 1989a) and Henriksson and Isacsson (2006) both found significant effects among women but not among men (with the exception of men over 60 years of age, among whom an effect was found in the intervention by Rutz et al.). Similarly, Szanto et al. (2007) found a significant decrease of suicide rates compared to the local control region for women only. Furthermore, there was a strong majority of female patients both being prescribed anti-depressant medication and undergoing treatment at the depression treatment clinic. These differences are however not consistent.
In the programme by Nutting et al. (2005), the effect was greatest amongst men, whilst Hegerl et al. (2006) found significant effects for both men and women. The remaining three interventions did not make a gender distinction in their data collection or analyses.

Table 2. Interventions targeting detection and prevention of depression at a primary health care level.

\section{Author, region, target population}

Gask et al. (2004) Liverpool and

Manchester, UK.

General population.

Kick (1999).

Colorado, US

Patients of internal

medicine residents.

Moreno et al. (2003).

Panama.

General population.

Scott et al. (2002).

North England.

General population.

Thompson et al. (2000).

Hampshire, UK.

General population

\section{Study design, intervention}

RCT. Training of GPs at two different locations for improving detection and management of depression.

RCT. Educational intervention on detection of depression given to internal medicine residents

Cohort, quasi-experimental. Educational programme regarding detection and manage-ment of depression for primary care nurses.

Cohort, quasi-experimental. Depression detection training, individual feed-back and revision of practice guidelines. Interventions at two different practices

RCT, clinical-practice guidelines and educational programme regarding depression detection.
Outcome measures

Detection of and improvements on depressive symptoms amongst patients and patient satisfaction with health service.

Rates of depression detected. Correlation between detection and severity of symptoms after intervention.

\section{Rates of depression} detected amongst patients. Improvements of care given measured four weeks after intervention.

Sensitivity and specificity of diagnoses of depression after intervention.

Sensitivity to diagnosis of depression measured after 6 weeks and after 6 months.
Results

No sign effect on patient outcomes (levels of depression). Detection of depression was sign at one location.

No sign improvements in detection rates compared to control group.

Sign increase in identification of depression as well as increased referrals to a doctor compared with a care as usual control group.

Practice A showed improved detection and management of depressive patients. Practice $B$ showed an increased sensitivity but a decreased specificity.

No sign effects on detection or outcomes compared with care as usual control group.

\section{Interventions targeting detection} and prevention of depression at a primary health care level

\section{General findings}

Five interventions focusing on improved detection of depression in a primary health care setting and fulfilling the other inclusion criteria were found.

One programme targeting detection of depression amongst 42 primary care nurses in Panama resulted in significantly increased rates of identification of depression and referrals to GPs, following a two-day seminar (total of 8 hours) (Moreno et al., 2003). However, these effects were seen amongst patients with moderate depression only and were absent for patients with more severe depression. No results regarding patient outcomes were reported. Kick (1999) carried out an educational intervention for residents of internal medicine, who were either in the first, second, or third year of study. During three monthly-occurring sessions the residents were informed on the
AHCPR Quick Reference Guide for depression in primary care.

Although a change in attitudes and knowledge regarding care of depressed patients was observed, no changes in the residents' ability to detect depression among patients were found. Similarly, no significant effects on the detection of depression or patients' outcome measures were achieved following an educational intervention consisting of 4 hours long seminar teaching through multimedia resources (such as video tapes) and the 
availability of educators for 9 months in a representative sample of sixty primary health practices in England (including 170 GPs) (Thompson et al., 2000).

This randomised controlled trial did include data on patient outcomes, but without any significant changes.

Gask and coworkers (2004) evaluated an educational intervention including GPs in the areas of Liverpool (20 participating GPs) and Manchester (18 participating GPs) in England. The GPs participated in a 10 hour training course which was carried out during 5 evenings. Some significant improvements in patients' own perceived health but not in depressive symptoms or in general health were found. With regard to the detection of depressive symptoms, significant improvements could be seen in Manchester but not in Liverpool. It should be noted that baseline experiences differed between the two settings, with GPs in Liverpool having more previous psychiatric, counselling and communication training than their colleagues in Manchester.

Another intervention resulting in divergent regional outcomes was published by Scott et al. (2002). This program was carried out in two clinics of areas with different socio-economic status in northern England. It involved individual feedback to GPs regarding their performance on a baseline measurement of sensitivity and specificity related to the identification of depression and anxiety among patients. Steering groups were also formed in both practices, whose task was to design practice-specific guidelines for treatment and referrals to counsellors. The results showed a clear difference in effect between the practices. Practice A (serving 10000 predominately white middle-class patients) increased the levels of detected cases of depression to near expectations (from 285 to 362, where the predicted number was 399) and successfully developed practice-specific guidelines. Practice B served 5500 patients within a deprived urban area and with a large proportion of ethnic minorities. Practice B also increased the levels of detected cases, the gap to the predicted number, however remained large (from 52 cases to 71 out of 208 predicted). The intervention in practice $B$ resulted in an increase in sensitivity, a decrease in specificity and the attempt to agree upon practice-specific guidelines failed.

\section{Discussion}

\section{General remarks}

Five out of the six articles which included suicide prevention as outcome measures reported preventive effects of suicidal ideation or suicidal behaviour. Still, in three studies these effects were limited to females and in one to patients with major depression. Two further articles (Pfaff et al., 2001 and Nutting et al., 2005) succeeded in improving the identification of suicidal ideation.

Results on interventions aiming solely to improve the detection of depression are somewhat more inconsistent. Two articles (Moreno et al., 2003; Scott et al., 2002) found improved identification of depression in patients. On the other hand, two further interventions (Gask et al., 2004; Scott et al., 2002) resulted in significant improvements in only one out of two different locations. Finally, the remaining two interventions (Kick, 1999; Thompson et al., 2000) did not result in any significant effects. Studies included in this review focussed on interventions primarily based on the general population seeking medical care in primary care settings. The findings have therefore to be interpreted respectively as we excluded studies focussing on specific treatment options and patient groups. Below follow discussions on certain aspects that might be of importance for an intervention's outcomes and success.

\section{Sustainability of effects}

In the literature reviewed here, there are some examples of poor sustainability of initial effects. For example, Bruce et al. (2004) report on significant effects of the intervention after four months, but not after eight or twelve months. Rutz et al. (2001) also found a decline in effectiveness during a longer follow-up period where the peak effect seemed to occur after two years of intervention. In 1988, five years after the initiation of the intervention, suicide rates had increased to just below baseline levels. It should be noted, however, that at this point in time half of the GPs that participated in the intervention were no longer working on the island. Nutting et al. (2005) also report on a decrease of the effects after a six-month period. The remaining interventions included here had either very short follow-up periods (e.g. Pfaff et al. 2001, follow-up after six weeks), or no follow-up at all (Henriksson and Isacsson, 2006; Hegerl et al., 2006; Szanto et al, 2007; Asarnow et al., 2005). Hence, no conclusions regarding the long-term effects of these interventions can be drawn. Based on these observations, repetitions of the educational programmes are likely to be beneficial to maintain the initially achieved effects.

\section{Needs assessments}

\section{Reasoning for using needs assessments}

In the health promotion literature the use of needs assessments is often highlighted as an important tool for designing effective interventions (see for example Tones \& Green, 2004; Naidoo \& Wills, 2000 for overviews). By considering what the health needs are in a particular area or for a particular population, resource allocation and prevention strategies can be optimised.

A general lack of needs-assessments prior to implementation, or at least the reporting of such, appeared from the literature reviewed. Only the Gotland study noted that the prevention activity originated in a request from the GPs themselves on education concerning the detection and treatment of depression (Rutz et al., 1989a, 1989b). This motivation of the GPs towards the educational programme might have contributed considerably to the effectiveness of the programme. The educational need was also noticeable due to the high suicide rates (well above the national average) compared to the low prescription rate of antidepressants (below the national average) as was also the case for the target-areas in the reports of Szanto et al. (2007) and Henriksson \& Isacsson (2006). Henriksson \& Isaksson (2006) evaluated the participants' perception of the intervention; $83 \%$ responded that the programme would be of benefit in their daily work and 63\% reported that they had gained new knowledge as a result of the programme.

Although Gask et al. (2004) did not describe a needs assessment as such, the results still allow for relevant interpretation. As discussed above, this intervention had a significant effect in Manchester but not in Liverpool, whilst on baseline 
measurements participating GPs in Liverpool had greater previous experience in psychiatry and training in counselling skills. Thus, although there were socioeconomic differences between the areas that might also have influenced the results, it is likely that the apparent discrepancy in outcome was partly due to variations in baseline levels of skills and knowledge of the participating staff. Thus, in the case of educational programmes directed to primary health care staff, it seems to be of importance to carefully assess the staff's baseline knowledge, attitudes and motivation towards an intervention before it is planned and carried out. This increases the likelihood that GPs are better prepared to take in new information and change their way of practice. Furthermore, it might be beneficial to investigate the patients' own suggestions for improvement in care as this might suggest important areas in need of improvements seen from a perspective otherwise easily missed.

\section{Gender differences}

In the literature reviewed considerable gender differences in the effects of the interventions were found. In three out of six reviewed studies the suicide preventive effect was only found for women. Discrepancies in findings with regard to gender have been reported considering the occurrence of suicidal behaviour and depression, help-seeking behaviour, symptom reporting, coping styles and even the clinical manifestation of depression (Rutz et al., 1989; Oliver et al., 2005; Angst et al., 2002). Depression in men, which may have different clinical features than depression in women, is suggested to be considerably underdiagnosed and under-treated (Rutz et al., 1989). This "male depressive syndrome" may be particularly characterised by acting-out, aggression, low impulse control and abuse or abuse equivalent behaviour among other characteristics (Rutz, 2001). "The Gotland Male Depression Scare" was designed to capture these characteristics (Rutz, 2001) and has since been validated and used as a screening instrument in primary health care (Zierau et al., 2002; Möller-Leimkuehler et al., 2004).
A follow-up intervention of the study conducted on Gotland was carried out in 1994 in which information regarding the "male depressive syndrome" was included both in the education material as well as in information campaigns directed to the public. Subsequent to this broader intervention approach, the suicide rates in men on Gotland decreased (Rutz et al., 1999).

Thus, attempts to improve the knowledge of health care staff regarding the male depressive syndrome, to facilitate male help-seeking and to change attitudes concerning help-seeking behaviour might be important tools in reducing suicide rates in men. These aspects have been taken into consideration in another study with significant decrease in male suicidality (Hegerl et al., 2006). Inclusion of arenas where males can be easily reached, like working places, may have an additional effect.

\section{Differences in methodological issues}

Although the interventions identified to match the inclusion criteria are divided into two groups (those including suicide prevention and those focusing on depression detection only), they are in fact very similar in design. The aim of all interventions was to improve the care of patients suffering from depression; what separates them is whether suicide prevention is included as an additional aspect. This is not only evident in the outcome measures used, but may also have been reflected in the educational contents. The lack of detailed descriptions of the actual activities comprising the interventions complicates both interpretation and comparison between interventions. Factors such as quality of teaching, teaching environment and content of the educational material might be crucial in the reception and effectiveness of the intervention.

The lack of detailed descriptions of the treatments offered further hinders the comparison of the mentioned studies focussing on patients' outcomes. If different types of therapies are provided along with the programmes, variation in the type of treatment rather than the education might underlie the discrepancies in effectiveness. A better description of these aspects in future preventive work is therefore warranted.
There are however a few characteristics for which there is an apparent difference between the interventions. One such characteristic is the time given to the intervention; while some interventions consisted of several days of education others only lasted a few hours. This is likely to have affected the depth of the education given and thus the effectiveness of the intervention. The participation rates also differed greatly between the studies. For interventions with low participation rates it is less likely that any actual effects will be apparent, as the impact on the whole population being studied will still be too small.

\section{The statistical dilemma}

Even if suicidal behaviour places a great burden on the affected individuals, their relatives and on the health care system, its occurrence is still rather rare, fortunately. This is, however, associated with considerable statistical problems, as a large amount of patients is necessary in an intervention project to assure a statistically significant change in rates of suicide and suicide attempt (Gunnell et al., 1994). One solution to overcome this obstacle could be to form multi-centre studies, like the "European Alliance against Depression" (Pfeiffer-Gerschel et al., 2007). The research network originates in the intervention carried out in Germany and Gotland (Hegerl et al., 2006; Rutz et al., 1989). It intends to gather information from several different European centres thereby increasing the total number of included subjects and subsequently the statistical power to detect a significant change in rates.

\section{Conclusion}

The results of the interventions reviewed were inconsistent, but still a number of important issues that might be crucial for a positive outcome emerged: First, needs assessments should be carried out prior to the planning and implementation of the intervention, the content of the intervention should then be adapted to the needs of the participating staff.

Second, gender differences in the clinical manifestation of depression and helpseeking behaviour should be considered in the design of the programmes. Third, the quality, length and type of educational programmes as well as the therapies 
provided should be carefully monitored and described in order to facilitate comparisons with other similar interventions. Fourth, multi-centre co-operations would be of benefit in order to strengthen the statistical power of the results. Finally, repetition of training is likely to increase the sustainability of the effects.

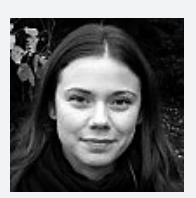

Stina K. Söderqvist has a master degree in health promotion from the Department of public health sciences, Karolinska Institutet, Stockholm. She is currently a PhD student in developmental cognitive neuro-science at the Department of woman and child health, also at KI.

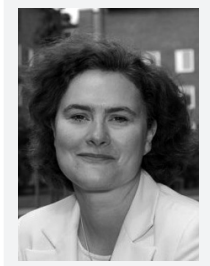

Ellenor Mittendorfer-Rutz holds a PhD in Psychosocial Medicine and is primarily engaged in suicide prevention programmes in Eastern European countries as well as epidemiological research on risk factors for suicidal behavior.

She is a researcher at the Department of clinical neuroscience, Section of personal injury prevention, Karolinska Institutet.

Correspondence to E. Mittendorfer-Rutz. Email: ellenor.mittendorfer-rutz@ki.se

\section{References}

Angst J, Gamma A, Gastpar M, Lepine J-P, Mendlewicz J, Tylee A. (2002). Gender differences in depression. Epidemiological findings from the European DEPRES I and II studies. European Archives of Psychiatry and Clinical Neuroscience, 252, 201-209.

Asarnow, J.R., Jaycox, L.H., Duan, N. et al. (2005). Effectiveness of a quality improvement intervention for adolescent depression in primary care clinics - A randomised controlled trial. Journal of the American Medical Association, 293, 311319.

Bruce, M.L., Ten Have, T. R., Reynolds, C.F. et al. (2004). Reducing suicidal ideation and depressive symptoms in depressed older primary patients - a randomised controlled trial. Journal of the American Medical Association 291, 1081-1091.

Gask, L., Dowrick, C., Dixon, C. et al. (2004). A pragmatic cluster randomized controlled trial of an educational intervention for GPs in the assessment and management of depression. Psychological Medicine, 34, 63-72.

Gunnell D, and Frankel S (1994). Prevention of suicide: Aspirations and evidence. British Medical Journal, 308, 1227-1233.
Hegerl, U., Althaus, D., Schmidtke, A. et al. (2006). The alliance against depression depression: 2 year evaluation of a community-based intervention to reduce suicidality. Psychological Medicine, $36,1225-1233$

Henriksson, S. and Isacsson, G. (2006). Increased antidepressant use and fewer suicides in Jämtland county, Sweden, after a primary care educational programme on the treatment of depression. Acta Psychiatrica Scandinavica 11, 159-167.

Kick, S.D. (1999). An educational intervention using the agency for health care policy and research depression guidelines among internal medicine residents. International Journal of Psychiatry in Medicine, 29, 47-61.

Luoma J.B., Martin C.E., Pearson J.L. (2002) Contact with mental health and primary care providers before suicide: a review of the evidence. American Journal of Psychiatry, 159, 909-916.

Lönnqvist, J.K., Henriksson, M.M, Isometsä, T. et al. (1995). Mental disorders and suicide prevention. Psychiatry and Clinical Neurosciences, 49, Suppl, 1, S111-116.

Lönnqvist, J.K. (2000). Psychiatric aspects of suicidal behaviour: depression. In The Handbook of Suicide and Attempted Suicide. (eds. K. Hawton and K. Van Heeringen). pp. 107-120. Chichester: John Wiley.

Mann, J.J., Apter, A., Bertolote, J. et al. (2005). Suicide prevention strategies: a systematic review. Journal of the American Medical Association, 294, 2064-2074.

Mittendorfer-Rutz E, Wasserman D. (2004). Trends in adolescent suicide mortality in the WHO European Region. European Child and Adolescent Psychiatry, 13, 321-331.

Mittendorfer-Rutz E, Wasserman D. (2004). The WHO European monitoring surveys on national suicide prevention programmes and strategies. Suicidologi , 9, 23-25.

Moreno, P., Saravanan, Y., Levav, I. et al. (2003). Evaluation of the PAHO/WHO training programme on the detection and treatment of depression for primary care nurses in Panama. Acta Psychiatrica Scandinavica, 108, 61-65.

Möller-Leimkühler, A.M., Bottlender, R., Strauß, A. et al. (2004). Is there evidence for a male depressive syndrome in inpatients with major depression? Journal of Affective Disorders, 80, 87-93

Naidoo, J., and Wills, J. (2000). Health promotion, foundations for practice. 2 nd edition. Elsevier Limited, London.

Nutting, P.A., Dickinson, L.M., Rubenstein, L.V. et al. (2005). Improving detection of suicidal ideation among depressed patients in primary care. Annals of Family Medicine, 3, 529-536.

Oliver MI, Pearson N, Coe N et al. (2005) Helpseeking behaviour in men and women with common mental health problems: cross-sectional study. British Journal of Psychiatry, 186, 297-301.
Pfaff, J.J., Acres, J.G., and McKelvevy, R.S. (2001). Training general practitioners to recognise and respond to psychologial distress and suicide ideation in young people. Medical Journal of Australia, 174, 222-226.

Pfeiffer-Gershel T,Wittman M, Hegerl U. (2007) "The European Alliance Against Depression". A European network targeting at effective care in community-based services for persons suffering from depressives disorders. Neuropsychiatrie:

Klinik, Diagnostik, Therapie und Rehabilitation, 21, 51-8.

Rutz, W. Wålinder, J., Eberhard, G. et al. (1989a). An educational programme on depressive disorders for general practitioners on Gotland: background and evaluation. Acta Psychiatrica Scandinavica, 79, 19-26.

Rutz,W., von Knorring, L., and Wålinder, J. (1989b). Frequency of suicide on Gotland after systematic postgraduate education of general practitioners. Acta Psychiatrica Scandinavica, 80, 151-154.

Rutz, W. (1999). Improvement of care for people suffering from depression: the need for comprehensive education. International clinical psychopharmacology, 14, 27-33.

Rutz W. (2001). Preventing suicide and premature death by education and treatment. Journal of Affective Disorders, 62, 123-129.

Scott, J. Thorne, A. and Horn, P. (2002). Effect of a multifaceted approach to detecting and managing depression in primary care. British Medical Journal, 325, 951-954.

SUPRE the WHO world wide initiative for the prevention of suicide. (2007). Live your life. [Online] Available from:

http://www.who.int/mental_health/management /en/SUPRE_flyer1.pdf [Accessed on 11 May, 2007]

Szanto, K., Kalmar, S., Hendin, H., Rihmer, Z., and Mann, J. (2007). A suicide prevention programme in a region with a very high suicide rate. Archives of General Psychiatry, 64, 914-920.

Tones, K., and Green, J. (2004). Health promotion: planning and strategies. Sage publications, London.

Thompson, C., Kinmonth, A.L., Stevens, L. et al (2000). Effects of a clinical-practice guideline and practice-based education on detection and outcome of depression in primary care: Hampshite Depression Project randomised controlled trial, Lancet 355, 185-191.

WHO (the World Health Organisation). (2007). Mental Health - Depression. [Online] Available from: http://www.who.int/mental_health/ management/depression/definition/en/index.html [Accessed on june 08]

Zierau F, Bille A, Rutz W, Bech P. (2002). The Gotland male depression scale: a validity study in patients with alcohol use disorders. Nordic Journal of Psychiatry, 56, 265-269. 